\title{
Between-side Comparisons of Iliotibial Band Flexibility and the Tibial Torsion Angle in Subjects with an Asymmetric Hallux Valgus Angle
}

\author{
Su-jung Kim, Ph.D. ${ }^{1}$; Kwang-jun You, Msc ${ }^{2}$; Do-young Jung, Ph.D. ${ }^{3}$ \\ ${ }^{1}$ KEMA Healing Center, Daejeon, South Korea \\ ${ }^{2}$ Samkwang Welfare Facilities, Iksan, South Korea. \\ ${ }^{3}$ Dept. of Physical Therapy, Joongbu University, Geumsan, South Korea.
}

Background Recent studies have used kinematic chain theory to establish a relationship between the hallux valgus and the lower limb joints and bones. This theory suggests that movement at one joint of a lower extremity may affect all other joints via a chain reaction.

Purpose We examined the hip joint range of motion and muscle strength in subjects with asymmetric hallux valgus angles.

Study design A cross-sectional study.

Methods Fifteen participants with asymmetric hallux valgus angles were recruited. Two examiners measured the hallux valgus, intermetatarsal and tibial torsion angles, and the iliotibial band flexibility. The independent $t$-test was used to compare the differences between the two sides.

Results The side with the greater hallux valgus angle also had a larger intermetatarsal angle. The iliotibial band was significantly less flexible on this side. The tibial torsion angles did not differ significantly between the two sides.

Conclusions We suggest that the hallux valgus angle is associated with iliotibial band flexibility. Therefore, stretching of the iliotibial band should be considered during rehabilitation of the hallux valgus.

Journal of KEMA

2018; 2(1): 11-15

Published Online

June 30, 2018

pISSN: 2586-4351

eISSN: 2586-5706

\begin{abstract}
Article History
Received 19 April 2018

Revised 26 April 2018

Accepted 30 April 2018
\end{abstract}

\section{CONTACT}

ptsports@joongbu.ac.kr

Do-young Jung,

Department of Physical

Therapy, College of Medi-

cal Science, Joongbu Uni-

versity, Geumsan-gun,

Chungnam, South Korea

This is an Open-Access article distributed under the terms of the Creative Commons Attribution Non-Commercial License (http://creativecommons. cense (ittp.//creativecommons. org/licenses/by-nc/4.0) which permits unrestricted non-commercial use, distribution, and reproduction in any medium, provided the original work is

Key words Hallux valgus; Iliotibial band; Tibial torsion.

\section{INTRODUCTION}

Hallux valgus (HV) is a progressive foot deformity characterized by lateral deviation of the first metatarsal creating an apex (a deformity) at the first metatarsalphalangeal joint, with or without medial soft tissue enlargement of the first metatarsal head (bunion). ${ }^{1,2,3}$ The prevalence of $\mathrm{HV}$ ranges from 12 to $70 \%$ in the general population. ${ }^{4,5}$ Nix et al. (2010) reported pooled prevalence estimates for $\mathrm{HV}$ of $23 \%$ in adults aged $18-65$ years and $35.7 \%$ in those aged $>65$ years. $^{6}$ HV may cause several health problems, changing the foot balance, triggering laxity of the static stabilizers (in turn disrupting muscle balance), and increasing the risk of falling. ${ }^{4,7,8}$

The metacarpophalangeal joint is dynamic, being affected by many muscles, joints, and ligaments. ${ }^{1,2}$ Most previous studies on HV focused on the possible relationship between the deformity and muscles and/or ligaments of the foot and lower leg. ${ }^{2,3}$ Recent studies used kinematic chain theory to link HV with the lower limb joints and bones. ${ }^{9,10}$ The theory suggests that movement at one joint of a lower extremity may affect all other joints via a chain reaction. ${ }^{2,3,9,10}$ A literature search for causes of $\mathrm{HV}$ did not produce any data on the general population. ${ }^{1,2,3}$ Also, no study has yet compared lower leg alignment and muscle flexibility between two sides in subjects with asymmetric HV angles (HVAs). We assessed 
iliotibial band flexibility and tibial torsion angle in subjects with asymmetric HVAs. We hypothesized that both parameters would differ between the legs.

\section{METHODS}

\section{Participants}

A pilot test was initially performed to determine the required sample size. A sample size was calculated based on a two-group t-test in $\mathrm{G}^{*}$ Power software package (version 3.1.9.2; Franz Faul, University Kiel, Germany). The power analysis used data on HVAs. A total of 14 subjects were required for a power of $80 \%(\alpha=0.05, \mathrm{~d}=0.98)$. We recruited 15 subjects with differences $>5^{\circ}$ in the HVA between the two legs (5 males and 10 females). The mean age was $33.0 \pm 10.3$ years, mean height $166.5 \pm 6.1 \mathrm{~cm}$, and mean weight $61.2 \pm$ $9.2 \mathrm{~kg}$. The investigators had 14 and 6 years of physiotherapy experience, respectively. The exclusion criteria were radiating pain, strain of any lower extremity muscle, and any joint contracture. Prior to the study, the principal investigator explained all procedures and all subjects signed informed consent forms.

\section{Measurement of the HVA}

The HVA was measured on radiographs at the intersection between the long axis of the first metatarsal and the proximal phalanx. ${ }^{1,2,3,5}$ The axis of the first metatarsal was drawn through points that proximally and distally bisected the shaft of the bone, and the axis of the proximal phalanx was drawn through the midpoints of the proximal and articular surfaces (Figure 1). ${ }^{1,2}$ Three measurements were made and the average was used in analysis.

\section{Measurement of the intermetatarsal angle}

The intermetatarsal angle was measured between the long

Table 1. Comparison of measurements on both legs

\begin{tabular}{cccc}
\hline Measurement & \multicolumn{2}{c}{ Mean \pm Standard deviation } & $p$ \\
\hline & $\begin{array}{c}\text { The greater } \\
\text { HVA side }\left({ }^{\circ}\right)\end{array}$ & $\begin{array}{c}\text { The less } \\
\text { HVA side }\left({ }^{\circ}\right)\end{array}$ & \\
\hline $\begin{array}{c}\text { Hallux valgus } \\
\text { angle* }\end{array}$ & $17.63 \pm 5.75$ & $12.40 \pm 4.89$ & 0.01 \\
$\begin{array}{c}\text { Intermetatarsal } \\
\text { angle* }\end{array}$ & $12.37 \pm 2.68$ & $9.87 \pm 2.45$ & 0.01 \\
$\begin{array}{c}\text { Tibial torsion } \\
\text { angle }\end{array}$ & $26.07 \pm 5.89$ & $21.58 \pm 6.56$ & 0.06 \\
$\begin{array}{c}\text { Iliotibial band } \\
\text { flexibility* }\end{array}$ & $7.47 \pm 3.56$ & $10.71 \pm 3.19$ & 0.01 \\
\hline
\end{tabular}

$* p<0.05$.

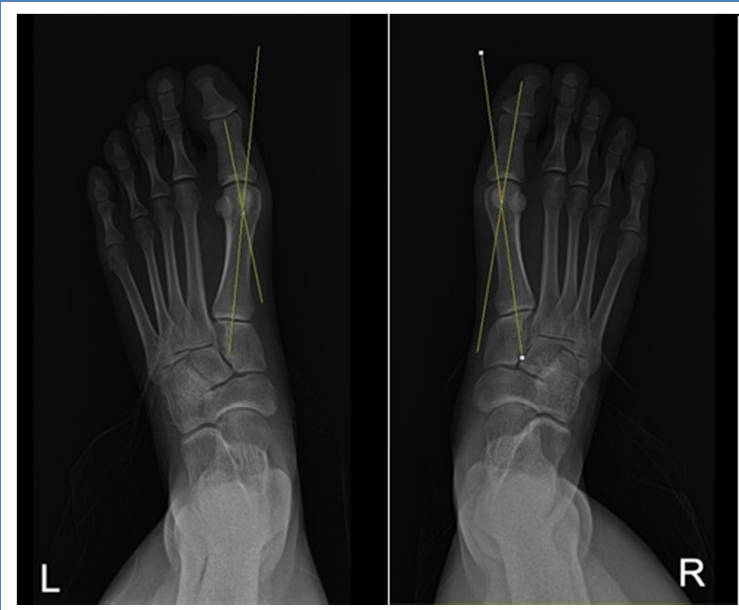

Figure 1. Measurement of hallux valgus angle.

axes of the first and second metatarsals (Figure 2). 1,2,3,5 Three measurements were made and the average was used in analysis.

\section{Measurement of the tibial torsion angle}

Each participant bent the knee through $90^{\circ}$ and held the ankle in the neutral prone position. The examiner located the middle of the medial and lateral malleolus (in the anteriorposterior direction) at the level of the ankle, and marked the midpoint with a pen. ${ }^{11}$ To derive the axis passing through both malleoli, a line connecting the points of the medial and lateral bones was drawn on the sole of the heel. The stationary and movable arms of the goniometer was placed in line with this axis and on the longitudinal axis of the femur, respectively (Figure 3). ${ }^{11}$ The examiner recorded the angle between the two lines as the tibial torsion angle. ${ }^{11}$ Each participant was instructed to remain relaxed during measurement to minimize rotation of the lower limb. Three measurements were made and the average was used in analysis.

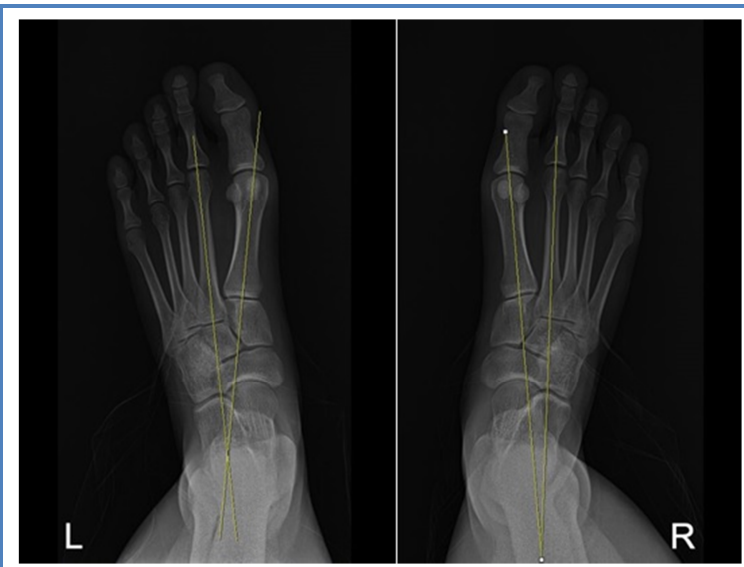

Figure 2. Measurement of intermetartasal angle 


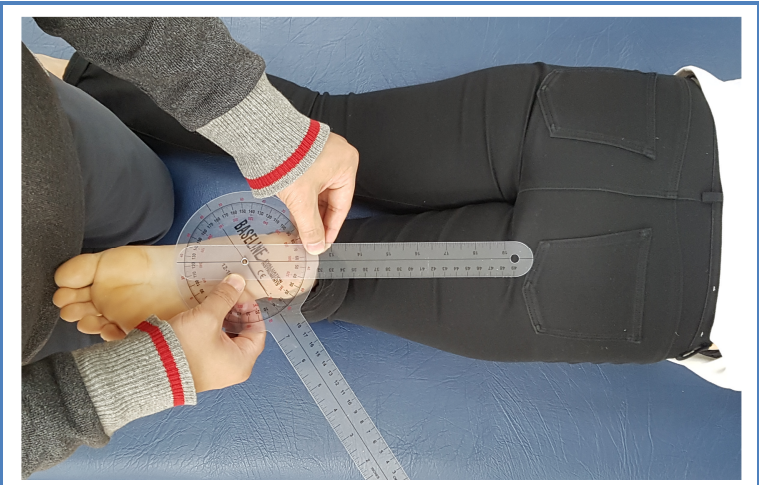

Figure 3. Measurement of tibial torsion angle.

\section{Measurement of iliotibial band flexibility}

We used the Ober test to assess iliotibial band flexibility. ${ }^{12}$ Each participant was instructed to bend the lower leg through $90^{\circ}$ to maintain the spine in a neutral position while lying on the side (Figure 4). The examiner stood behind the participant and placed a stabilizing hand on the upper iliac crest. The hip joint of the upper leg was placed in abduction and extension and the knee joint flexed through $90^{\circ}$. The examiner recorded the adduction angle with the upper leg pointing to the floor to a certain extent, ensuring that the pelvis did not move. Three measurements were made and the average used in analysis.

\section{Procedure}

Using anterior and posterior radiographs, obtained by employing the same protocol in the same hospital, the first examiner measured the HVA and intermetatarsal angle. The second examiner measured the tibial torsion angle and iliotibial band flexibility while blinded to the leg with the greater HVA. The first examiner collected all data and performed all analyses.

\section{Statistical analysis}

We used the independent $t$-test to compare differences in

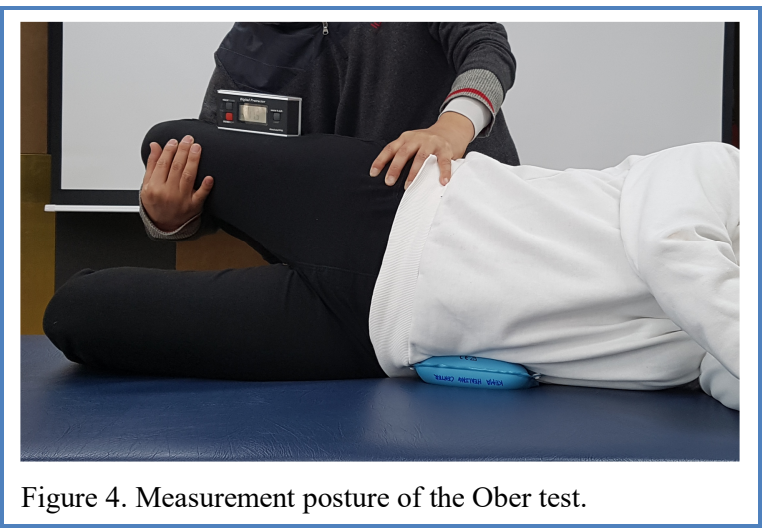

the HV, intermetatarsal, and tibial torsion angles, and in iliotibial band flexibility, between the two sides. The level of statistical significance was set to 0.05. SPSS software (ver. 12.0; SPSS, Inc., Chicago, IL, USA) was employed for all statistical analyses.

\section{RESULTS}

The HV and intermetatarsal angles differed significantly between the two sides (both $p<0.05$ ). Iliotibial band flexibility was significantly lower on the side with the greater HVA $(p<0.05)$. The tibial torsion angle did not differ significantly between the two sides $(p>0.05)$.

\section{DISCUSSION}

Earlier studies explored whether lower extremity alignment affected HV. ${ }^{2,13,14}$ We sought a link between asymmetric HVAs and lower extremity alignment. Iliotibial band flexibility differed significantly between the two sides. The causes of HV vary widely. ${ }^{2,6,15}$ Yamada et al. (2014) reported that a patient with a valgus deformity of the hindfoot also exhibited an HV deformity, and suggested that correction of any severe valgus, the calcaneal lateral offset, and any pronated foot deformity was required for HV rehabilitation. ${ }^{15}$ Foot pronation is caused by malfunction of muscles connected to the adjacent joint, and is closely associated with both adduction of the hip joint and internal rotation of the knee joint. ${ }^{16,17,18}$ Previous studies reported that these movements of proximal segments which is associated with excessive foot pronation was caused by predominance of tensor fascia lata during walking or stair-climbing. ${ }^{17,18}$ Then, although the amount of foot pronation was not measured in this study, it can be expected that asymmetric foot pronation may effect on the difference of iliotibial band flexibility in subjects with asymmetric HVAs.

In this study, the tibial torsion angles differed somewhat between the two sides but the difference was not significant, possibly because of the low numbers of subjects. We conducted a power analysis to determine the proper sample size before the study. Although the sample size was determined by 14 , it was required 25 subjects to explore a possible difference in the tibial torsion angle, when performing the power analysis with results of this study. If we increase the number of subjects, there is a risk of increasing "type 1 errors". Therefore, current study is proper in number of subjects. Thus, we suggest that asymmetry of HVA influences more IT band flexibility than tibial torsion angle.

Our study had several limitations. First, this was a static study; we do not know whether dynamic status differed and, 
if so, how. The body forms a connected mechanical chain. ${ }^{2,3,13,16} \mathrm{HV}$ causes not only foot problems, but also general malalignment and muscle strength imbalance in the lower extremities. ${ }^{2,3,4}$ The entire exercise chain requires study, i.e., not only the knee joint, but also the hip. Second, because subjects with normal as well as mild HVA participated in this study, the results of this study cannot be generalized in clinical setting for HV rehabilitation. Our inclusion criterion was a between-foot difference $\geq 5^{\circ}$. Previous studies classified HV into three types by the HVA and intermetatarsal angle: normal $\left(\mathrm{HVA}<15^{\circ}\right.$, intermetatarsal angle $<9^{\circ}$ ) mild (HVA 15-20 intermetatarsal angle $10-15^{\circ}$ ), moderate (HVA $21-40^{\circ}$, intermetatarsal angle $15-20^{\circ}$ ), and severe (HVA $>40^{\circ}$, intermetatarsal angle $\left.>20^{\circ}\right) .{ }^{19,20}$ The HVAs of the feet of our subjects were $17.63 \pm 5.75^{\circ}$ and $12.40 \pm 4.89^{\circ}$, respectively. The intermetatarsal angles were $12.37 \pm 2.68^{\circ}$ and $9.87 \pm 2.45^{\circ}$, respectively. To generalize in clinical setting for HV rehabilitation, further work is required to determine whether iliotibial band flexibility and tibial torsion differ among subjects normal and with HV groups in three stages.

\section{CONCLUSION}

Subjects with asymmetric HVAs exhibited asymmetric knee joint musculoskeletal elements. Each lower extremity forms a kinematic chain, and any abnormal joint alignment triggers misalignment and asymmetry of adjacent joints and muscles. Therefore, we suggest that iliotibial band flexibility should be considered in HV rehabilitation.

\section{Key Points}

Question Do between-leg differences in iliotibial band flexibility and tibial torsion angle affect the hallux valgus angle?

Findings We found a significant difference in iliotibial band flexibility between the two sides in subjects with asymmetric hallux valgus angles.

Meaning Iliotibial band flexibility may be associated with the hallux valgus angle.

\section{Article information}

Conflict of Interest Disclosures: None.

Funding/Support: None.

Acknowledgment: None.

\section{REFERENCES}

1. Glasoe WM, Nuckley DJ, Ludewig PM. Hallux valgus and the first metatarsal arch segment: A theoretical biomechanical perspective. Phys Ther. 2010;90:110-120.
2. Steinberg N, Finestone A, Noff M, Zeev A, Dar G. Relationship between lower extremity alignment and hallux valgus in women. Foot Ankle Int. 2013;34(6):824-831.

3. Steinberg N, Siev-Ner I, Zeev A, Dar G. The association between hallux valgus and proximal joint alignment in young female dancers. Int J Sports Med. 2015;36(1):6774.

4. Menz HB, Lord SR. Gait instability in older people with hallux valgus. Foot Ankle Int. 2005;26:483-489.

5. Roddy E, Zhang W, Doherty M. Prevalence and associations of hallux valgus in a primary care population. $A r$ thritis Rheum. 2008;59:857-862.

6. Nix S, Smith M, Vicenzino B. Prevalence of hallux valgus in the general population: A systematic review and meta-analysis. J Foot Ankle Res. 2010;3:21.

7. Wen J, Ding Q, Yu Z, Sun W, Wang Q, Wei K. Adaptive changes of foot pressure in hallux valgus patients. Gait Posture. 2012;36(3):344-349.

8. Perera AM, Mason L, Stephens MM. The pathogenesis of hallux valgus. J Bone Joint Surg Am. 2011;93:16501661.

9. Oatis CA. Kinesiology-the mechanics \& pathomechanics of human movement. Baltimore, MD: Williams \& Wilkins; 2009.

10. Sharma L, Song JS, Felson DT, et al. The role of knee alignment in disease progression and functional decline in knee osteoarthritis. JAMA. 2001;286:188-195.

11. Milner CE, Soames RW. A comparison of four in vivo methods of measuring tibial torsion. Journal of Anatomy. 1998;193:139-144.

12. Park JH, Kang SY, Choung SD, Jeon HS, Kwon OY. Effects of tibial rotation on Ober's test and patellar tracking. Knee. 2016;23(4):600-603.

13. Ohi H, Iijima H, Aoyama T, Kaneda E, Ohi K, Abe K. Association of frontal plane knee alignment with foot posture in patients with medial knee osteoarthritis. $B M C$ Musculoskelet Disord. 2017;18(1):246.

14. Van Gheluwe B, Kirby KA, Hagman F. Effects of simulated genu valgum and genu varum on ground reaction forces and subtalar joint function during gait. $J$ Am Podiatr Med Assoc. 2005 Nov-Dec;95(6):531-541.

15. Yamada S, Hirao M, Tsuboi $\mathrm{H}$, et al. Involvement of valgus hindfoot deformity in hallux valgus deformity in rheumatoid arthritis. Mod Rheumatol. 2014;24(5):851854.

16. Yoon KS, Park SD. The effects of ankle mobilization and active stretching on the difference of weight-bearing distribution, low back pain and flexibility in pronatedfoots subjects. J Exerc Rehabil. 2013;9(2):292-297.

17. Lack S, Barton C, Malliaras P, Twycross-Lewis R, 
Woledge R, Morrissey D. The effect of anti-pronation foot orthoses on hip and knee kinematics and muscle activity during a functional step-up task in healthy individuals: a laboratory study. Clin Biomech (Bristol, Avon). 2014;29(2):177-182.

18. Lack S, Barton C, Woledge R, Laupheimer M, Morrissey D. The immediate effects of foot orthoses on hip and knee kinematics and muscle activity during a functional stepup task in individuals with patellofemoral pain. Clin
Biomech (Bristol, Avon). 2014;29(9):1056-1062.

19. Coughlin MJ, Saltzman CL, Anderson RB. Mann's surgery of the foot and ankle. $9^{\text {th }} \mathrm{ed}$. Philadelphia, PA: Mosby; 2014.

20. Nery C, Coughlin MJ, Baumfeld D, Ballerini FJ, Kobata $\mathrm{S}$. Hallux valgus in males--part 2: radiographic assessment of surgical treatment. Foot Ankle Int. 2013;34(5): 636-644. 